\title{
Elevating the future of mobility UAV-enabled Intelligent Transportation Systems
}

This paper was downloaded from TechRxiv (https://www.techrxiv.org).

LICENSE

CC BY 4.0

SUBMISSION DATE / POSTED DATE

$18-10-2021 / 21-10-2021$

\section{CITATION}

Saboor, Abdul; Coene, Sander; Vinogradov, Evgenii; Tanghe, Emmeric; Joseph, Wout; Pollin, Sofie (2021): Elevating the future of mobility UAV-enabled Intelligent Transportation Systems. TechRxiv. Preprint. https://doi.org/10.36227/techrxiv.16826743.v1

$\mathrm{DOI}$

10.36227/techrxiv.16826743.v1 


\title{
Elevating the future of mobility: UAV-enabled Intelligent Transportation Systems
}

\author{
Abdul Saboor, Sander Coene, Evgenii Vinogradov, Emmeric Tanghe, Wout Joseph, Sofie Pollin
}

\begin{abstract}
Intelligent Transportation Systems (ITS) improve traffic efficiency, traffic management, driver's comfort, and safety. They consist of a broad range of components, including vehicles, sensors, Base Stations, Road Side Units, and road infrastructure (i.e., traffic signals). ITS of the near future will need to support multi-modal transportation schemes (including ground and aerial vehicles, so-called Urban Air Mobility). ITS will have to be integrated with Unmanned Aerial Systems Traffic Management (UTM) and rely on 3 Dimensional (3D) connectivity provided by Integrated Aerial-Terrestrial 6G networks to achieve this support. In other words, various types of Unmanned Aerial Vehicles (UAVs) will become integral parts of future ITS due to their mobility, autonomous operation, and communication/processing capabilities. This article presents our view on the future integration of ITS and UTM systems, enabling wireless technologies and open research questions. We also present how UAVs can be used to enhance the performance of the currently available ITS.
\end{abstract}

\section{INTRODUCTION}

$\mathbf{T}$ He last century observes a significant rise in urbanization with more people migrating to urban areas. This influx of new residents increases the size of cities around the globe. Cities are being forced to address traffic congestion caused by an increased demand for transporting people and goods to support the population. Moreover, the growing number of single-person households and longer life expectancy are causing demand to shift towards individual mobility services that can be adapted to users' specific life circumstances. Currently, the mobility sector is undergoing huge changes due to several technological revolutions and paradigm shifts. Socalled Mobility 2.0 includes i) driver-less cars; ii) cooperation between vehicles (e.g., supported by $5 \mathrm{G}$ networks), iii) electric cars to reduce pollution, and iii) shared individual transport (e.g., cars, bikes or scooters). However, there is another Revolution that is underway already.

Urban Air Mobility (UAM) envisions a safe and efficient transportation system that will use highly automated aircraft operating and transporting passengers or cargo at lower altitudes within urban and suburban areas. In May 2021, Morgan Stanley released a prediction [1] stating that by 2050 the total market of UAM (delivery, air taxi, patrolling drones, to name a few) will reach up to $\$ 19$ tn (10-11\% of projected global Gross Domestic Product (GDP)). Fig. 1 shows the expected technology development timeline [1]. Drone delivery will become part of urban life within the next ten-fifteen years,

Abdul Saboor, Evgenii Vinogradov, and Sofie Pollin are with Department of Electrical Engineering, KU Leuven, Belgium

Sander Coene, Wout Joseph and Emmeric Tanghe are with Department of Information Technology, IMEC-Ghent University, 9052 Ghent, Belgium.

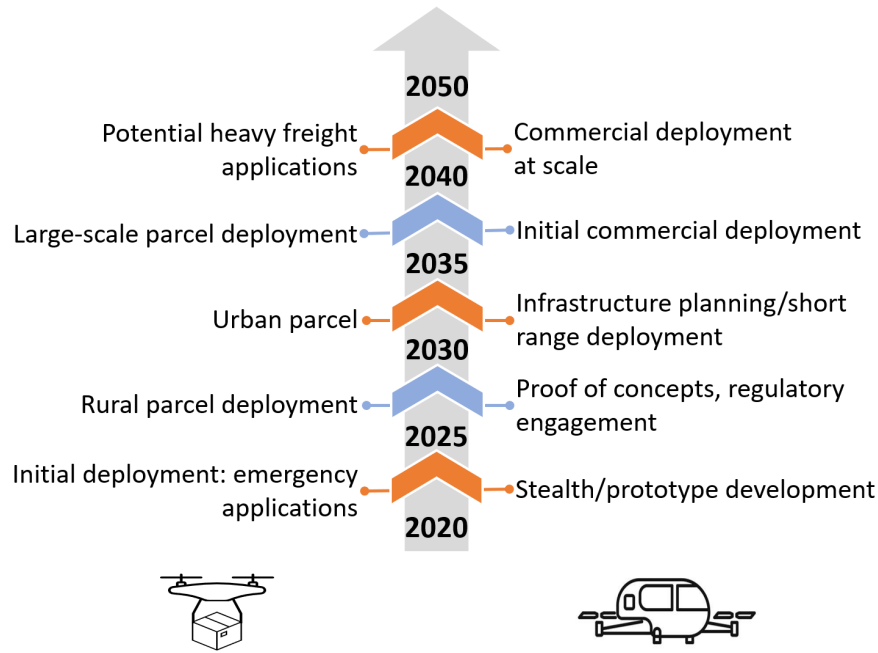

Fig. 1. UAM technology development timeline.

commercial introduction of urban passenger aircraft will take around 20-30 years.

Interestingly, the same 3 Dimensional (3D) shift is observed in the telecommunication sector [2]. One of the $6 \mathrm{G}$ enablers (expected to be in place around 2030) is Integrated GroundAir-Space Networks, including drones, satellites, high altitude platforms, and ground infrastructure. These two 3D trends reenforce and help each other creating new use cases and attracting a lot of attention from academia and industry. Indeed, the technological innovations we seek now should be brought to another dimension: autonomous ground vehicles become autonomous Unmanned Aerial Vehicles (UAVs); electric cars become electric Vertical Take-Off and Landing (eVTOL) aircraft; to ensure cooperation, we need an ubiquitous 3D communication. Considering that the ground vehicles will not disappear, can we already talk about a new paradigm of Mobility 3.0 (or rather Mobility 3D)?

Of course, first, we have to think about the missing technologies for Mobility 2.0. For example, the Intelligent Transportation System (ITS) is a crucial element. However, while selecting the tools to achieve the goals, we should keep future challenges in mind. Shall we already start including UAVs in ITS? Luckily, in 2017, Menouar et al. showed in [3] that using drones for ITS are highly beneficial. In this article, we answer two questions: how can we use drones for ITS in the near future, and what technologies will be needed for Mobility 3D. 


\section{OVERVIEW INTELLIGENT TRANSPORTATION SyStemS}

Conceptually, ITS aims to reduce friction for participants in a transportation scenario. At the end of the last century, ITS saw independent development in countries like the USA, China, Japan, and countries inside of Europe, each spearpointing different priorities. Early examples include navigation, which combines Global Positioning System (GPS) (or any other Global navigation satellite system (GNSS)) with a digital roadmap to provide real-time instructions to get to a set destination. These ITS also contain automated toll systems using Radio Frequency Identification (RFID) chips or license plate recognition systems to identify cars and reduce the time needed for the payment. In addition, induction coils underneath the road count the passing vehicles and inform public infrastructure spending by identifying traffic bottlenecks.

As the technology evolved, so has ITS. The main area where one can observe a significant improvement in wireless communications. This paradigm shift is reflected in the new term: Connected ITS (C-ITS). In the last decades, many sensors have been added to the arsenal of inputs, and new ways of influencing transportation have been added as outputs. For example, Closed-Circuit Television (CCTV) cameras can now not only count the number of vehicles but also track the vehicle types, congestion forming, visibility conditions, and accidents. Dynamic road signs can warn vehicles in advance of adverse road or traffic conditions, imposing suitable speed limits. Realtime tracking of buses and trams provides users with up-todate travel information like arrival times and occupancy on mobile apps or information signs. Similarly, public transport capacity can be dynamically adjusted throughout the day by tracking the number of passengers. Local weather forecasts and friction sensors can inform city services to deploy snowplowing vehicles or take anti-icing measures.

It has been shown that road users are more likely to respond and comply with hyper-local recommendations rather than more widely applicable warnings (e.g., weather conditions mentioned on dynamic road signs vs. over the radio). Such hyper-local communication is the goal of Vehicle-to-Vehicle networks, to relay highly relevant information in the direct vicinity with minimal lag and high priority. While such protocols and network topologies have been investigated and implemented, they are not readily available in contemporary cars. Additionally, inter-fleet communication is impossible when traffic is more dispersed than the communication range limitations support.

As we mentioned in the introduction, driver-less cars are important new agents of transportation systems. However, the next generation ITS is not limited to the automation of vehicles only. In contrast, it requires automating the infrastructure, supporting units, devices, and network. An ITS combines sensors, computers and communication technologies for efficient traffic management. The ITS incorporates Vehicleto-everything (V2X) communication to enhance traffic efficiency. The communication between vehicles, infrastructure, pedestrians, and devices allows them to share the information for different services such as congestion control, collision avoidance, traffic light control, and parking.

\section{Next Generation Intelligent 3D TRANSPORTATION SYSTEM}

Authors of [3] demonstrated that UAVs help ITS from the wireless communications point of view because of mobility and better/stable radio channel. In this section, we describe several ways of using UAVs to assist ITS. However, following the predictions presented in [1], we start with describing drones as new agents of the transportation systems of the future.

\section{A. Sky is the Limit: Urban Air Mobility}

The transportation paradigm shifts towards 3D mobility will drastically change the traffic patterns, demand and required infrastructure. In the following, we provide examples of the potential applications.

1) Cargo Drones:

a) Emergency: Small drones can carry necessary medications or blood. A study conducted by American Heart Association shows that exposure to traffic increases the chances of a cardiac arrest. The traffic caused approximately $8 \%$ of cardiac arrest cases. In this case, a UAV can quickly deliver an Automated External Defibrillator (AED) that helps restart the heart rate.

b) Road Congestion-friendly eCommerce: With the rise in online purchasing behavior, traffic congestion caused by delivery vehicles has increased as well. As a result, research is being done on reducing the traffic load by using drones as part of the delivery system. Both UAV-only and truck-plus-UAV have already been studied as improvements. A step further makes use of the existing fixed route transportation systems, like public buses and trains. They provide a (moving) recharging point where the drone can rest in-between deliveries.

c) Temporary ITS Infrastructure: UAV can act as a temporary sign, i.e., variable speed limit and traffic lights at a particular area. The acquired data can be analyzed using machine learning algorithms to examine and predict traffic behavior in the presence of various signs. Finally, the fixed infrastructure (signs, roads) will be installed in suitable locations. One more important advantage of adding an extra UAV layer to ITS is the ability to rapidly provide supporting material to remote positions, including fuel, aid, or toolkit.

\section{2) People Transportation:}

a) Air Taxis: Commercial UAM operations will help to avoid transportation congestion on the ground. Around the world, there are companies currently providing these services. For example, BLADE Bounce is an on-demand helicopter transfer service between BLADE helipad locations and New York area airports—both commercial and private. Similarly, Airbus is operating its Voom on-demand helicopter service in Sao Paulo, Brazil. However, the new all-electric and hybrid eVTOL promise to be more accessible and efficient. These vehicles are currently being designed by Boeing, Uber, Airbus, Hunday, and other companies.

b) Emergency: In addition to passenger-carrying flights, UAM also includes aircraft operations for tasks in metropolitan areas such as public safety, medical evacuations and rescue. 


\section{B. Bird-eye View: UAV as a Flying Eye}

The majority of commercially available UAV-based solutions are exploiting on-board cameras. This is rather a mature technology which is used (up to some extent) in ITS. However, the "flying eye" is still a growing market.

a) Emergency: UAVs can reach an accident location fast and analyze the situation's severity using visual information. Furthermore, UAVs store and analyze the traffic information in its map. Therefore, it can re-route the ambulance to the emergency spot using the optimal route.

An interesting approach will be the drone-assisted ambulance service. In this scenario, a drone moves ahead of the ambulance, controlling the traffic and signals using cellular technology to minimize the congestion for the ambulance. Generally, an ambulance worker/driver has to drive fast that increases the ambulance crew's life risk. Therefore, an autonomous system is more suitable for emergency services. Also, the road-based emergency support is not efficient as compared to the aerial support.

b) Law Enforcement: Police are an essential part of the ITS. The key duties of police include protection, law enforcement, patrolling, violation tracking and others. UAVs can help the police to enforce rules with a better/broader view than traditional police vehicles. For example, Chinese police use UAVs to instruct a bike rider to put the helmet on. Similarly, the UAV as a police unit tracks the traffic violations such as speed, wrong direction, and safety parameters. As a result, UAV identifies the vehicle and later help the department in issuing a traffic challan.

Because of better speed and visibility, a UAV is beneficial in chasing scenarios. First, UAVs keep track of criminal vehicles and continuously inform police about the actual whereabouts of such vehicles. Second, UAVs track the vehicle number and suspect images to distribute them to different police stations/units. A UAV can also fly ahead of the suspected car and limit its movability by directly controlling or indirectly controlling the traffic signals. Last, UAVs reach crime scenarios and provide criminal details, i.e., the number of criminals and weapons.

c) UAV-aided Traffic Monitoring, Conflict Detection:

Due to the bird-eye view on the scenery and cameras working in different parts of the spectrum, UAVs enable safer road use by pedestrians, cyclists, and Powered Two-Wheeler riders (PTW: motorcycles, mopeds, etc.), collectively referred to as Vulnerable Road Users (VRU). According to World Health Organization, VRUs represent the majority of road traffic fatalities with 23\%, 22\%, and 5\% of deaths for PTW, pedestrians, and cyclists, respectively. The factors affecting VRU safety demonstrated high socio-economic dependency (e.g., the majority of causalities occur in urban/rural environments in developed/developing countries). However, several common features are noted: the most dangerous maneuver is a road crossing, and the most dangerous time of the day is twilight or darkness. This is explained by worsened visibility and driver's blind spots.

d) ITS Infrastructure inspection: UAVs add an additional layer of supportability to the traditional ITS. The traffic signal and signs provide necessary information to the commuters

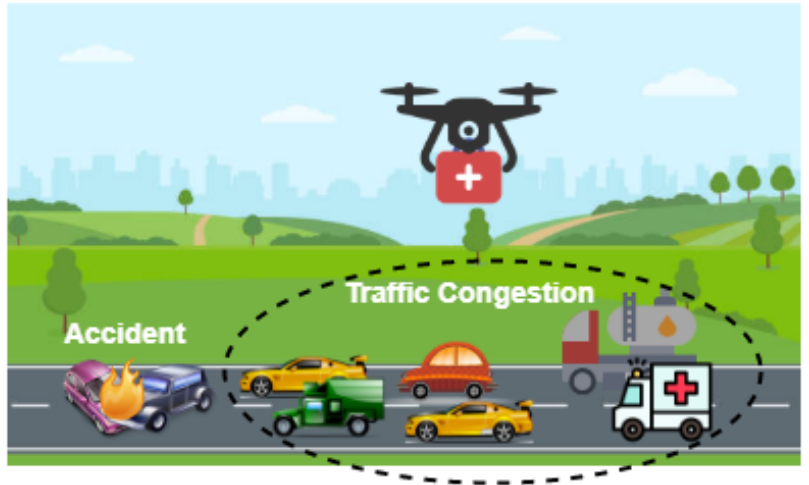

a) Urban Air Mobility

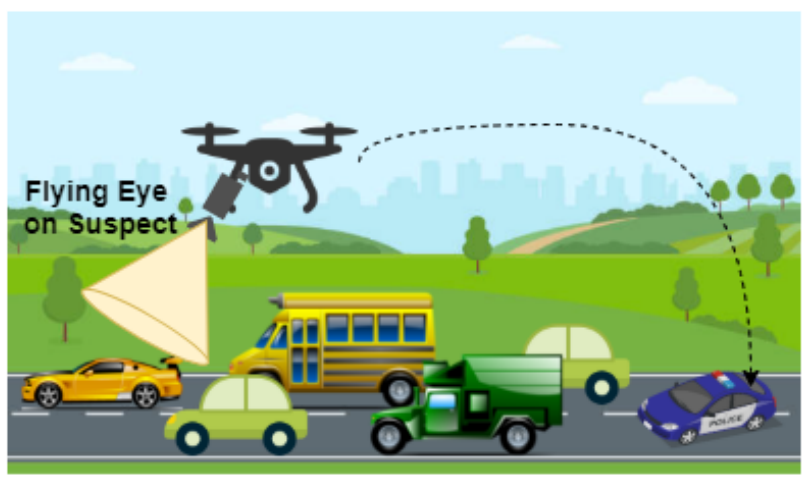

b) UAV as a Flying Eye

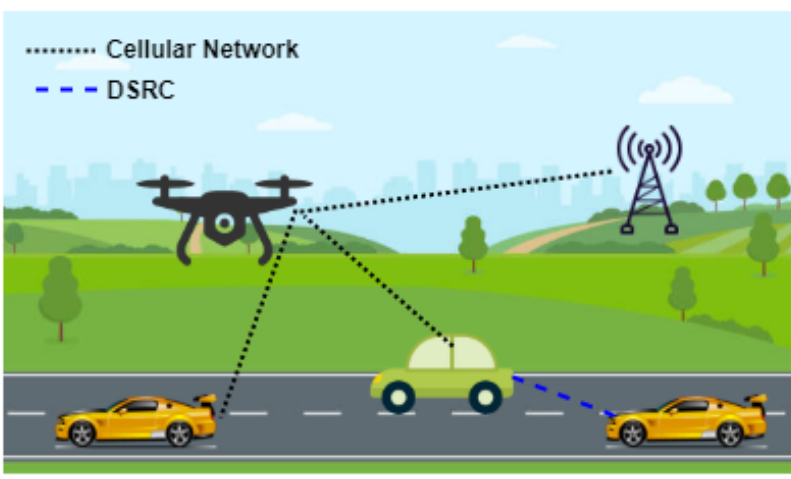

c) UAV-aided Connectivity

Fig. 2. UAV use cases in ITS: a) a UAV is providing early medication by skipping traffic congestion, b) a UAV is chasing a suspect and sending information to the police, c) a UAV is acting as ABS to provide connectivity in road vicinity.

and vehicles. Traffic signalization is critical, especially in the case of autonomous vehicles. However, the negative impact on signalization can affect the performance of the ITS. It includes damaged traffic signs, wrong directions, blurry information, or poor retroreflective markings. Generally, this damage requires a visual analysis, and UAVs can help because of their movability. For example, a UAV analyzes the parameters such as pole's serial number, material, height, sign, dimensions of sign, sign's code, and light color. Later, It can signal the required information to the designated unit that can then take action. Furthermore, a UAV connects to Geographic Information System (GIS) database and takes measurements 
such as dimensions and height of signal.

e) Public Service: Like public transport route inspection, the roadside conditions are monitored more efficiently with a fleet of drones. Information on drive-ability (snow, inundation) is transmitted and updated regularly to relevant authorities. Additionally, daily inspection of powerlines, bridge structures, and pavement decreases the risk of sudden catastrophic public property failure.

UAVs have been successfully used to make 3D scans of buildings. They can be used to follow up construction works (both public and private), spotting unsafe situations, and providing better estimates for completion time, leading to better city planning overall. Start-to-end route analysis, realtime and full-scale load observation on roads leads to better navigation planning and provide clues for city planning where additional lanes or roads are needed.

\section{Help From the Skies: UAV-aided Connectivity}

C-ITS enable vehicles to interact with each other and with the surrounding road infrastructure involving communication between vehicles using V2V, V2X, and I2I communications. The benefits of C-ITS span a range of areas (e.g., including improving road safety and optimizing transport efficiency). Despite a significant body of work in this area, vehicular communication in the urban environment is still severely affected by the harsh propagation environment (e.g., dynamic obstacles). UAVs can offer a more stable communication link due to the flight altitude, ensuring persistent line-of-sight channel [4].

a) Flying Roadside Unit (RSU): RSU is an indispensable part of ITS and the RSU distribution directly influences vehicular communications. However, intermittent connectivity, high routing overhead, inflexible communication infrastructure, and unscalable architecture are the key challenges that severely hinder the wide applications of vehicular networks. Overcoming these hurdles is possible via using UAV-mounted RSUs due to their inherent features like more stable channel (Line of Sight (LoS) link over longer distances) and dynamic on-demand deployment.

b) Aerial Base Stations (ABSs): Recently, the trend of relying on cellular networks for ITS support is gaining a lot of attention. For example, 5G Automotive Association (5GAA, a global, cross-industry organization of companies from the automotive, technology, and telecommunications industries) indicates 5G as the next key technology for ITS. Indeed, cellular networks are able to ensure reliable communication between vehicles and infrastructure. However, fixed terrestrial infrastructure faces the same challenges (though at a lower scale) as fixed RSU deployment. Logically, the idea of putting a base station on a drone has appeared.

A relevant overview of $\mathrm{ABS}$ deployment techniques is provided in [4]. Note that up to our best knowledge, the literature is still lacking an analysis of ABS usage for vehicular communications.

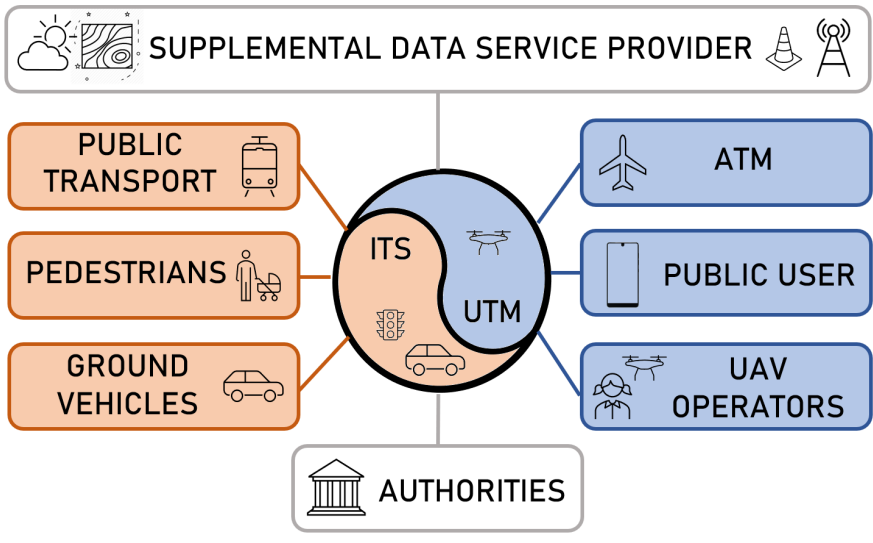

Fig. 3. Converging UTM and ITS for next generation 3D ITS

\section{Key Technological Enablers For NeXT GENERATION ITS}

\section{A. Unmanned Air Systems (UAS) Traffic Management}

UAS Traffic Management (UTM) systems aim at providing a range of services to drone operators (both private and companies), authorities, and other air control bodies such as conventional Air Traffic Management (ATM).

The main functions of UTM are to i) strategically organize the airspace ii) ensure the cooperation of unmanned and manned aviation through a link to ATM systems iii) ensure transparency of drone operations to public users(citizens) iv) control the air traffic through flight permissions v) dynamically adapt drone operations depending on the circumstance (weather conditions, available telecommunication capabilities, etc.).

Bauranov and Rakas recently published an overview [5] of UTM activities performed by academia, industry, and national and supra-national airspace agencies (e.g., UTM solutions by Nanyang Technological University in Singapore, Amazon and Airbus, NASA and national airspace agencies as well as the European U-Space concept). Unfortunately, the authors did not include the view of the International Civil Aviation Organization (ICAO) on the recommended common UTM framework with core principles for global harmonization presented in [6].

We envision that coordination between ITS and UTM will be needed in the near future (see Fig. 3). Aerial and ground vehicles will need to synchronize their operation for optimizing the capacity of the complete 3D transportation network to balance the load of aerial and terrestrial traffic based on the weather or currently available capacity (e.g., changing due to the works on the road network). Novel solutions will need to be designed for 3D logistics, including aerial and ground vehicles. At the strategic level, the ITS, UTM, and Supplemental Data Service Providers (SDSP, providing communication services, GIS data or information about current micro-weather conditions, etc.) planners have to join the efforts in order to optimize their capital and operational expenditure while constructing the required infrastructure such as road networks, vertiports, operational centers, communication networks, etc. 


\section{B. Edge/fog}

Vehicles in ITS are equipped with several sensors and computing units, GPS, and mobiles. These devices communicate and transmit data (e.g., BSMs) using various protocols, such as Wireless Application Protocol (WAP) or Next-Generation Telematics Protocol (NGTP). Cloud computing is a viable solution to store and process this massive data for decision making. However, the low-latency requirements of vehicular communication become a bottleneck.

The introduction of fog and edge computing between the cloud and ITS components can overcome these limitations by bringing the storage, networking, and computing capabilities near ITS objects (i.e., vehicles). Moreover, it was demonstrated in [7], fog computing helps in overcoming the limited on-board computational power even for such a demanding use case as the processing of visual information from the "Flying eye."

RSUs act as fog units in the traditional ITS. However, RSU has geographical limitations that generate communication holes in the less dense RSU areas. Furthermore, a fixed RSU cannot provide its computing services due to unstable dynamic channel. Furthermore, a few places such as bus stops and university areas have a few peak hours, thus not requiring a permanent RSU. Therefore, UAVs will act as fog nodes to provide on-demand computation and communication in the above scenarios due to mobility. One primary concern for UAVs is power consumption during this process. However, while acting as a fog node, a UAV will land and provide on-demand processing. Therefore, it will result in reducing the power wastage due to hovering. Other creative ways to overcome this problem include UAV-assisted distributed fog computing [8].

\section{Artificial Intelligence}

Artificial Intelligence (AI) brings intelligence to machines using its sub-modules, i.e., Machine Learning (ML), Federated Learning (FL), and Reinforcement Learning (RL). Therefore, AI can act as an enabler for the next generation ITS by bringing intelligence to drones. For example, ML can help deploy optimal number ABSs in a specific area with maximum coverage. Furthermore, ML models can predict the wireless traffic Congestion and deploy ABS by minimizing their mobility. Similarly, ML algorithms can help in channel estimation between UAV-to-UAV or UAV-to-Ground. ML and RL also help in predicting the optimal trajectory of UAVs and detecting nearby UAVs to avoid collisions. FL is helpful in scheduling and resource allocation. For example, distributed Support Vector Machine (SVM)-based FL can determine the LAP or HAP each vehicle or user should connect. In addition, FL coupled with blockchain shows promising results in crowdsensing and mitigating security and privacy threats [9].

\section{D. $6 G$ networks: Integrated Aerial-terrestrial communication}

a) Channel modeling: A city is an environment with ever-changing number of users, their locations, and bandwidth needs. On the other hand, drones are highly dynamic mobile access points. To optimize deployment strategies and UAV payloads, more information is needed on the signal propagation between the user on the ground and the flying base station. There currently exist a number of channel models that address wireless propagation in an urban environment. An overview of those models can be found in [4], [10]. No channel models consider UAV-to-vehicle communication which is vital for UAV-enabled ITS. Moreover, the available models are only applicable in a limited frequency band, focus on fixed base stations and/or non-mobile end-users. Furthermore, it cannot address medium-altitudes (above buildings, up to several $\mathrm{km}$ ), a possible drone deployment space.

A model that addresses these shortcomings can lead to a better understanding of the fading effects between buildings and therefore assess the best drone deployment areas. Additionally, link stability between mobile BSs and mobile users (i.e., vehicles), can inform the best drone load-out and antenna designs, as well as the number of drones that need to cater to a given number of vehicles. And finally, the link loss for multi-hop infrastructure can be much more accurately modeled, yielding better infrastructure topology design.

b) Routing Algorithms: The vehicles are equipped with the On-Board Units (OBUs), including different transceivers and sensors. OBUs help the vehicles to communicate with BSs, vehicles, and RSUs. In general, Dedicated Short Range Communication (DSRC) is used for V2V and V2I communication. The routing in vehicles $(\mathrm{V} 2 \mathrm{~V})$ is essential for sharing critical messages, such as BSMs. Similarly, communication provides external services, such as internet access and route discovery. The vehicles form Vehicular Ad hoc NETwork (VANET) to transmit information in ITS wirelessly. But, the VANET poses several limitations for an efficient the ITS, including routing overhead, high packet collision, delay, scalability, and unreliable communication.

Unreliable communication is a significant challenge in ITS due to the highly dynamic nature of vehicles. For example, the change in V2V or V2I distance fluctuates the Received Signal Strength Indicator (RSSI) and results in Pathloss [11]. Similarly, the objects (buildings/trees) block the radio signals, causing Pathloss. All these factors make the communication unreliable, cause packet failures that lead to accidents.

Additionally, the V2I and V2V routing is done via multihopping reactively or proactively. The route creation or maintenance due to the dynamicity of vehicles generates a large routing overhead. Similarly, numerous handovers are required in V2I communication that causes extra overhead. One more problem with VANET in the ITS is its fixed infrastructure. Therefore, it is hard to extend its coverage or provide dynamic support in unexpected dense scenarios. Lastly, scalability is a concern because vehicles generate many packets, leading to network congestion, delays, and packet loss.

The UAV resolves the above problem because it is less prone to channel impairments due to better LoS. Therefore, it provides a reliable connection as compared to the traditional communication in ITS. Furthermore, UAVs will act as a relay to transmit messages from one vehicle to another because of their better coverage. This significantly reduces the routing overhead for sending the message over several cars. Therefore, UAV improves routing, scalability, and Packet Delivery Ratio 


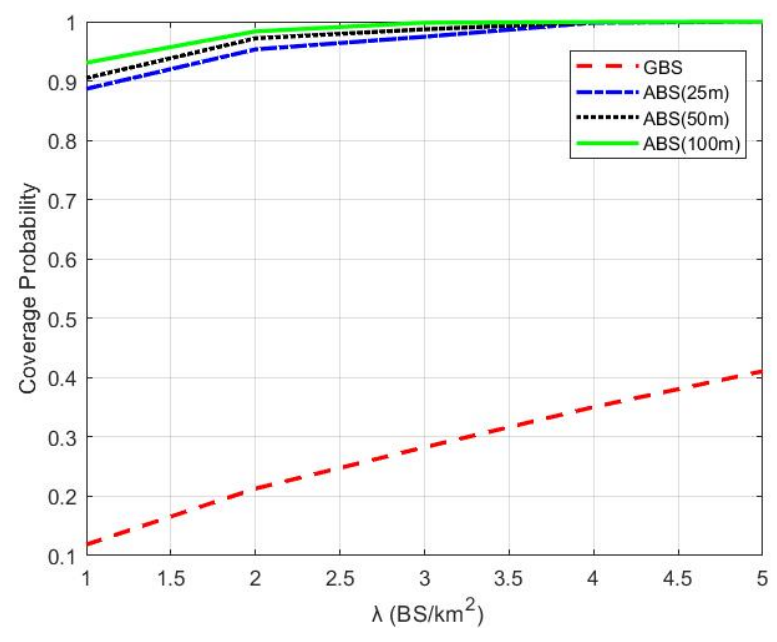

Fig. 4. Coverage probability as a function of $\lambda(\chi=1 \mathrm{~km})$.

(PDR).

c) Space-air-ground integrated networks: Current communications infrastructure is primarily focused on users at ground level. Near-future visions of intra- and inter-city transportation include eVTOLs, which would require the communications network to extend up in the air as well [12].

Space-Air-Ground Integrated Networks promises a vision of highly flexible and dynamic network infrastructure that relies on the integration of satellites, High- and Low-Altitude Platforms (HAP/LAPs), tethered balloons, UAVs, and terrestrial base stations to provide unprecedented coverage, from ground to high-altitude. Such coverage foresees the use of UAVs not only as an end-user but also as a chain in the network itself. An integrated network aims to bridge the gap between the distinct advantages (and disadvantages) of each platform type. While satellites have good coverage overall, their limited throughput, receiver needs, and signal delay limit their usability for certain real-time applications but excel at multicasting information to a large group. High-altitude platforms provide a cost-effective solution to service a larger area than base stations and reduce the number of handovers required for fast-moving vehicles. UAV-based stations will bridge large distances by achieving line-of-sight positions and can be easily and dynamically deployed but cannot stay in the air indefinitely.

\section{CASE STUdY: TERRESTRIAL AND AERIAL BASE STATIONS FOR VEHICLE COMMUNICATIONS}

In stochastic geometry, the Poisson Point Process (PPP) is a popular approach to model the position of wireless nodes in a defined space/area with intensity $\lambda$ [13]. Therefore, this paper places the ABSs and Terrestrial/Ground Base Stations (GBS) in a circular space with radius $\chi$. However, the PPP is not always suitable for positioning vehicles because their location is restricted to roadways. Therefore, this case study uses a Poisson Line Process (PLP) to model random roads in the same area with intensity $\Lambda$ [14].

This case study considers 200 cars randomly distributed over the lines/roads defined by the PLP. Furthermore, it
TABLE I

SPECTRAL EFFICIENCY AS A FUNCTION OF HEIGHT. GBS AND ABS ARE POSITIONED AT CENTER OF POISSON AREA WITH $\chi=0.5 \mathrm{KM}$

\begin{tabular}{|c|c|c|}
\hline Base Station & Height $(\mathrm{m})$ & Spectral Efficiency $(\mathrm{Bit} / \mathrm{s} / \mathrm{Hz})$ \\
\hline GBS & 15 & 7.60 \\
\hline \multirow{3}{*}{ ABS } & 40 & 12.43 \\
\cline { 2 - 3 } & 60 & 12.35 \\
\cline { 2 - 3 } & 80 & 12.30 \\
\cline { 2 - 3 } & 100 & 12.29 \\
\hline
\end{tabular}

assumes 5G ABS and GBS with perfect beamforming, hence completely mitigating the impact of interference. It is also assumed that the transmission and receiver antenna gain is constant $\left(G_{t x}=10 \mathrm{dBi}, G_{r x}=2 \mathrm{dBi}\right)$ and each car will be served by the closest base station. The other parameters are: transmission power $\left(P_{t x}=23 \mathrm{dBm}\right)$, frequency $(f=3.5 \mathrm{GHz})$ and bandwidth $(\beta=20 \mathrm{MHz})$. The Coverage Probability $(C P)$ and Cumulative Distribution Function $(C D F)$ of the Signal to Noise Ratio (SNR) are computed over 500 realizations.

This case study uses the Urban Macro (UMa) path loss model from the 3rd Generation Partnership Project [15]. Fig. 4 shows the $C P$ against varying $\lambda$ for GBSs and ABSs at the heights of $25 \mathrm{~m}, 50 \mathrm{~m}$, and $100 \mathrm{~m}$. The locations of 200 vehicles, ABSs, and GBSs are randomly generated with the PPP and PLP using $\chi=1 \mathrm{~km}$ and $\Lambda=2$. The $C P$ is defined according to the SNR threshold $(\delta=30 \mathrm{~dB})$. The UAV or ABS has a better LoS probability than GBS due to the channel geometry [4]. Due to the better channel, ABSs provide better coverage than GBS deployed with the same density. Simulations show that ABSs can achieve more than $95 \% C P$ compared to $35 \% C P$ for $\lambda=4 \mathrm{BS} / \mathrm{km}^{2}$. In contrast, 35 GBSs are required to achieve $95 \% C P$ with $\delta=30 \mathrm{~dB}$.

Fig. 5 shows the CDF as function of SNR. The locations of vehicles, ABSs, and GBSs are randomly generated with PPP and PLP using $\chi=2 \mathrm{~km}$ and $\Lambda=2$ to assess the performance/coverage over a wider area. As the LoS probability is directly proportional to height, the highest ABS at $100 \mathrm{~m}$ (ABS100) shows better mean SNR than at $50 \mathrm{~m}$ (ABS50), at $25 \mathrm{~m}$ (ABS25), and GBS. However, the height also contributes to the Pathloss. As a result, the peak SNR can only be achieved by the lower base stations. Therefore, GBS and ABS25 offer higher SNRs than ABS50 and ABS100 for a small proportion of vehicles placed close to these stations.

Finally, Table [I illustrates the Spectral Efficiency $(S E)$ against the ABS height. In contrast to previous results, this simulation considers one GBS and ABS placed in the center of the defined area with $\chi=0.5 \mathrm{~km}$ and $\Lambda=2$, without PPP. However, the roads and vehicle locations are determined according to PLP. Results indicate that ABS at height $40 \mathrm{~m}$ shows $63.5 \%$ more $S E$ than GBS. The $S E$ reduces slightly with altitude due to larger Pathloss, as shown in Table I]

\section{CONCLUSION}

The next generation ITS will have to rely on $3 \mathrm{D}$ connectivity provided by Integrated Aerial-Terrestrial 6G networks. It brings UAVs as an essential part of the ITS system. This paper envisions the convergence of UTM and traditional ITS for the next generation 3D ITS. Therefore, this paper lists 


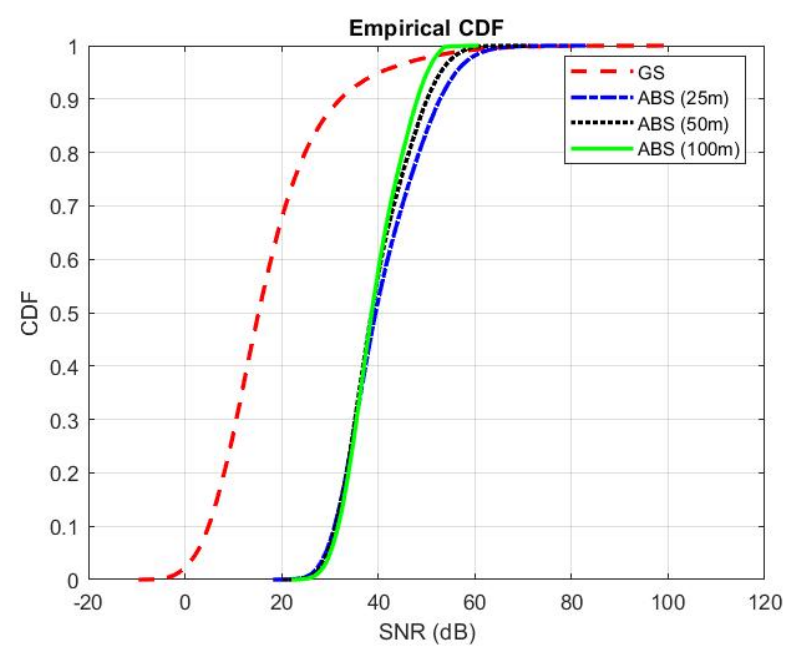

Fig. 5. Cumulative Distribution Function (CDF) of SNR using GBS and $\operatorname{ABS}(\mathrm{h}=25 \mathrm{~m}, 50 \mathrm{~m}, 100 \mathrm{~m})$.

drones as new agents of ITS and describes different use cases. Furthermore, this paper discusses the key for the full deployment of next generation UAV-enables ITS. Lastly, a case study is presented to compare the $S E, C P$, and $S N R$ of ABS and GBS. Results show that $95 \%$ coverage is achieved using approximately 4 ABS compared to $35 \%$ coverage using the same intensity of GBS. Furthermore, ABSs provide a better mean SNR and show $63.5 \%$ more $S E$ over GBS.

\section{ACKNOWLEDGMENT}

This research is supported by the Research Foundation Flanders (FWO), project no. G098020N.

\section{REFERENCES}

[1] A. Jonas, R. Shanker, K. Liwag, M. Sharpe, B. Kovanis, E. Silverberg, E. Zaghi, and M. Pasterchick, "eVTOL/Urban Air Mobility TAM Update: A Slow Take-Off, But Sky's the Limit," Morgan Stanley Research, May 2021.

[2] G. Geraci, A. Garcia-Rodriguez, M. Azari, A. Lozano, M. Mezzavilla, S. Chatzinotas, Y. Chen, S. Rangan, and M. D. Renzo, "What Will the Future of UAV Cellular Communications Be? A Flight from 5G to 6G," arXiv:2105.04842v1, 2021.

[3] H. Menouar, I. Guvenc, K. Akkaya, A. S. Uluagac, A. Kadri, and A. Tuncer, "UAV-Enabled Intelligent Transportation Systems for the Smart City: Applications and Challenges," IEEE Communications Magazine, vol. 55, no. 3, pp. 22-28, 2017.

[4] E. Vinogradov, H. Sallouha, S. De Bast, M. Azari, and S. Pollin, "Tutorial on UAVs: A Blue Sky View on Wireless Communication," Journal of Mobile Multimedia, vol. 14, no. 4, pp. 395-468, 2018.

[5] A. Bauranov and J. Rakas, "Designing airspace for urban air mobility: A review of concepts and approaches," Progress in Aerospace Sciences, vol. 125, p. 100726, 2021. [Online]. Available: https://www.sciencedirect.com/science/article/pii/S0376042121000312

[6] International Civil Avation Organization, "Unmanned Aircraft Systems Traffic Management (UTM) - A Common Framework with Core Principles for Global Harmonization - Edition 3," Tech. Rep., 2021.

[7] Q. Chen, H. Zhu, L. Yang, X. Chen, S. Pollin, and E. Vinogradov, "Edge Computing Assisted Autonomous Flight for UAV: Synergies between Vision and Communications," IEEE Communications Magazine, vol. 59, no. 1 , pp. 28-33, 2021.

[8] G. Lee, W. Saad, and M. Bennis, "Online Optimization for UAV-Assisted Distributed Fog Computing in Smart Factories of Industry 4.0," in 2018 IEEE Global Communications Conference (GLOBECOM), 2018, pp. 16.
[9] Y. Wang, Z. Su, N. Zhang, and A. Benslimane, "Learning in the Air: Secure Federated Learning for UAV-Assisted Crowdsensing," IEEE Transactions on Network Science and Engineering, vol. 8, no. 2, pp. 1055-1069, 2021.

[10] P. S. Bithas, V. Nikolaidis, A. G. Kanatas, and G. K. Karagiannidis, "UAV-to-Ground Communications: Channel Modeling and UAV Selection," IEEE Transactions on Communications, vol. 68, no. 8, pp. 51355144, 2020.

[11] A. Raza, S. H. R. Bukhari, F. Aadil, and Z. Iqbal, "An UAV-assisted VANET architecture for intelligent transportation system in smart cities," International Journal of Distributed Sensor Networks, vol. 17, no. 7, p. 15501477211031750, 2021

[12] N. Saeed, T. Y. Al-Naffouri, and M.-S. Alouini, "Wireless communication for flying cars," Frontiers in Communications and Networks, vol. 2 p. 16, 2021.

[13] H. ElSawy, A. Sultan-Salem, M.-S. Alouini, and M. Z. Win, "Modeling and analysis of cellular networks using stochastic geometry: A tutorial," IEEE Communications Surveys Tutorials, vol. 19, no. 1, pp. 167-203, 2017.

[14] V. V. Chetlur and H. S. Dhillon, "Coverage analysis of a vehicular network modeled as cox process driven by poisson line process," IEEE Transactions on Wireless Communications, vol. 17, no. 7, pp. 44014416, 2018.

[15] 3GPP, "Enhanced LTE support for aerial vehicles," Technical Report 36.777, Jan 2018

Abdul Saboor is working towards his $\mathrm{PhD}$ at the Department of Electrical Engineering, KU Leuven. His research aims at UAVs and WBANs.

Sander Coene is working towards his $\mathrm{PhD}$ at IMEC-Ghent University. His research aims channel modeling.

Evgenii Vinogradov obtained his Ph.D. degree in 2017 from UCLouvain. Currently, Evgenii is working on $6 \mathrm{G}$ wireless communications and UAVs in KU Leuven. He actively participated in SESAR (Single European Sky ATM Research) project Percevite.

Emmeric Tanghe has been a Postdoctoral Researcher in propagation modeling with the Department of Information Technology at Ghent University (imec-UGent/INTEC) since 2011. In October 2015, he became a part-time professor in medical applications of electromagnetic fields.

Wout Joseph is professor in the domain of experimental characterization of wireless communication systems with Ghent University. He has been an imec PI, since 2017. His research work dealt with measuring and modeling of electromagnetic fields around base stations for mobile communications related to the health effects of the exposure to electromagnetic radiation.

Sofie Pollin is professor at KU Leuven focusing on wireless communication systems. Her research centers around wireless networks that require networks that are ever more dense, heterogeneous, battery powered and spectrum constrained. She pioneered a $5 \mathrm{G}$ testbed for distributed Massive MIMO at $\mathrm{KU}$ Leuven, and is now leading the way towards $6 \mathrm{G}$ tests in multiple large EU projects. 